\title{
TITLE:
}

\section{Analysis and Compensation of Transmitter IQ Imbalances in OFDMA and SC-FDMA Systems}

\section{$\operatorname{AUTHOR}(\mathrm{S})$ :}

Yoshida, Yuki; Hayashi, Kazunori; Sakai, Hideaki; Bocquet, Wladimir

\section{CITATION:}

Yoshida, Yuki ... [et al]. Analysis and Compensation of Transmitter IQ Imbalances in OFDMA and SC-FDMA Systems. IEEE Transactions on Signal Processing 2009, 57(8): 3119-3129

\section{ISSUE DATE:}

2009-08

URL:

http://hdl.handle.net/2433/204387

\section{RIGHT:}

(C) 2009 IEEE. Personal use of this material is permitted. Permission from IEEE must be obtained for all other uses, in any current or future media, including reprinting/republishing this material for advertising or promotional purposes,

creating new collective works, for resale or redistribution to servers or lists, or reuse of any copyrighted component of this work in other works.; This is not the published version. Please cite only the published version.; この論文は出版社版

でありません。引用の際には出版社版をご確認ご利用ください。 


\title{
Analysis and Compensation of Transmitter IQ Imbalances in OFDMA and SC-FDMA Systems
}

\author{
Yuki Yoshida, Student Member, IEEE, Kazunori Hayashi, Member, IEEE, Hideaki Sakai, Fellow, IEEE, and \\ Wladimir Bocquet, Member, IEEE
}

\begin{abstract}
1 Abstract-One limiting issue in implementing high-speed wireless systems is the impairment associated with analog processing due to component imperfections. In uplink transmission of multiuser systems, a major source of such impairment is IQI introduced at multiple transmitters. In this paper, we deal with OFDMA and SC-FDMA which have received attention in recent years as physical layer protocol in WiMAX and 3GPP LTE, and analyze the effect of the transmitter ( $T x)$ IQ imbalances on OFDMA and SC-FDMA receivers. To cope with the inter-user interference problem due to Tx IQ imbalances, we propose a widely linear receiver for OFDMA and SC-FDMA systems and also propose a novel subcarrier allocation scheme, which has high tolerance to such Tx IQ distortion.
\end{abstract}

Index Terms-IQI, OFDMA, SC-FDMA, IFDMA

\section{INTRODUCTION}

\section{A. Introduction}

Recently, orthogonal frequency division multiplexing (OFDM) [1] based multiple access schemes are attracting much attention for uplink physical layer protocols in highspeed wireless networks. The most prominent example of this is orthogonal frequency division multiple access (OFDMA) employed in IEEE802.16e (WiMAX) [2]. Other significant example is single carrier frequency division multiple access (SCFDMA) [3], including interleaved frequency division multiple access (IFDMA) [4], which results from the application of discrete Fourier transform (DFT) preceding to the modulation process in OFDMA. Due to the low peak-to-average-powerratio (PAPR) of the modulated signals, SC-FDMA can eases the requirement on the power amplifiers of the transmitters. Therefore SC-FDMA has been employed for uplink physical layer protocol in 3GPP Long Term Evolution (LTE) [5], where lower PAPR greatly benefits mobile handsets in terms of power consumption.

A low-cost implementation of such physical layers is desirable in view of mass deployment, but challenging due to impairments associated with the analog components. A major source of analog impairments in high-speed wireless communications systems is the In-phase/Quadrature-phase imbalance (IQI) [6], [7]. The IQI is the mismatch between I and Q balances due to the analog imperfection and introduced both in the up- and down- conversion at the transceivers. In general, it is difficult to efficiently and entirely eliminate such imbalances in the analog domain due to power consumption, size and cost

\footnotetext{
${ }^{1}$ Copyright (c) 2008 IEEE. Personal use of this material is permitted. However, permission to use this material for any other purposes must be obtained from the IEEE by sending a request to pubs-permissions @ ieee.org.
}

of the devices. Therefore, efficient compensation techniques in the digital baseband domain are needed for the transceivers [8].

In this paper, we start with investigating the impact of IQIs introduced at the multiple transmitters in OFDMA and SCFDMA uplinks. Firstly, we describe the base band received signal models for the systems with general, interleaved and localized subcarrier allocations in the presence of transmitter (Tx) IQIs and also derive user-by-user received signalto-interference ratio (SIR) after user separation, where the interference is due to the Tx IQIs. Through our formulations, it will be revealed that the Tx IQIs sometimes cause severe interuser interference (IUI) at the receiver and the appearance of the IUI uniquely determined by the employed subcarrier allocation, e.g., in the OFDMA system with interleaved subcarrier allocation, the IUIs occur between certain pairs of users, while specific two users can avoid such IUI. Heretofore the analysis on the impact of Tx and/or receiver (Rx) IQIs and some useful compensation methods have been reported for the OFDM receivers [8]-[14]. However, to the best of our knowledge, few works has been done focusing on the such unfair IUI problem due to the Tx IQIs in OFDMA and SC-FDMA systems. Based on the analysis, we consider signal processing techniques to compensate for such distortions in OFDMA and SC-FDMA receivers. For the OFDM systems, the widely linear (WL) filtering approach [16], [17], which jointly elaborate the received signal and its conjugate, have been explicitly or implicitly adopted to the equalization problem in the presence of IQI. We also resort to the WL approach and propose WL equalization and multi-user detection method for OFDMA and SC-FDMA based on the zero-forcing (ZF) and minimummean-square error (MMSE) criteria. Especially for the system with interleaved subcarrier allocation, we propose an efficient pairwise equalizer which resulting from our analysis on the user dependent IUI. Finally, we propose a novel subcarrier allocation method, which has high tolerance to the Tx IQ distortions. Recalling, the subcarrier allocation dependent IUI component is the main source of performance degradation caused by the Tx IQIs. Therefore, by employing some specific subcarrier allocations, we can avoid the IUI and efficiently suppress the ill effect of the Tx IQIs at the OFDMA receiver.

This paper is organized as follows. Firstly, we introduce some matrices and their characteristic which will be used in the rest of the paper. Sec. II formulates the effect of Tx IQIs on the received OFDMA and SC-FDMA symbols. In Sec. III, the joint channel equalization and multi-user detection methods in the presence of Tx IQIs are proposed. Then we propose the novel subcarrier allocation method to avoid the 
IUI problem due to the Tx IQIs for OFDMA and SC-FDMA transmitters in Sec. IV. In Sec. V, the validity of our analysis and the effectiveness of the proposed methods are evaluated via computer simulations. Conclusions are given in Sec. VI.

\section{B. Notations and Groundwork}

The notations used in this paper are as follows: the usual bold capital letters are used to denote column vectors or matrices and $(\cdot)^{*},(\cdot)^{\mathrm{T}}$, and $(\cdot)^{\mathrm{H}}$, are complex conjugate, transpose, and Hermitian transpose of $(\cdot)$ respectively. An $M \times M$ identity matrix is denoted by $\mathbf{I}_{M}$ and $\mathbf{0}_{M \times N}$ is an all-zero matrix of size $M \times N$. We also use $\operatorname{diag}\{\mathbf{x}\}$ as a diagonal matrix whose diagonal elements consist of the vector $\mathbf{x},\|\cdot\|$ denotes the Euclidean norm of vector, and $\mathrm{E}[\cdot]$ is the expectation operation.

Further on, let us introduce some matrices and their properties which will be used in the paper. A $K M \times K M$ matrix W represents $K M$-point DFT matrix and its $(m, n)$ element is given by

$$
\mathbf{W}(m, n)=\frac{1}{\sqrt{K M}} e^{-j \cdot 2 \pi \frac{(m-1)(n-1)}{K M}},
$$

where $m, n=1,2, \cdots, K M$. On the other hand, $\overline{\mathbf{W}}$ denotes $M \times M$ DFT matrix. We also consider the square of the DFT matrix:

$$
\mathbf{W}^{2}(m, n)= \begin{cases}1 & (m=n=1 \text { or } m=K M-n+2) \\ 0 & \text { (otherwise) }\end{cases}
$$

where $m, n=1, \cdots, K M$. Thus $\mathbf{W}^{2}$ is a permutation matrix.

$\mathbf{U}$ is defined as $K M \times M$ expander matrix, whose $K m$-th row is equal to the $m$-th of $\mathbf{I}_{M}$ and the other rows are zero vectors. Left multiplication of $\mathbf{U}$ to a matrix represents an expand operation,i.e., inserting $K-1$ all zero rows between two neighboring rows of the matrix and increase the number of rows $K$ times. On the other hand, left multiplication of $\mathbf{U}^{\mathrm{H}}$ corresponds to decimation, which is the operation of picking up every $K$-th row. It can be verified that $\mathbf{U}$ can be expressed by using DFT matrix as [20]:

$$
\mathbf{U}=\frac{1}{\sqrt{K}} \mathbf{W}^{\mathrm{H}}\left[\begin{array}{c}
\mathbf{I}_{M} \\
\vdots \\
\mathbf{I}_{M}
\end{array}\right] \overline{\mathbf{W}}=\frac{1}{\sqrt{K}} \mathbf{W}\left[\begin{array}{c}
\mathbf{I}_{M} \\
\vdots \\
\mathbf{I}_{M}
\end{array}\right] \overline{\mathbf{W}}^{\mathrm{H}}
$$

We also introduce a $K M \times K M$ down-shifting matrix $\Pi$ whose $(m, n)$ elements $\Pi(m, n)$, with $m, n=1, \cdots, K M$, is given by

$$
\boldsymbol{\Pi}(m, n)= \begin{cases}1 & (m=n+1 \\ 0 & \text { (otherwise })\end{cases}
$$

Left multiplication of $\Pi$ denotes the column wise circular down-shift operation. Since the shifting matrix is a circulant matrix, we can diagonalize it using DFT matrix as

$$
\begin{aligned}
\boldsymbol{\Pi} & =\sqrt{K M} \mathbf{W}^{\mathrm{H}} \operatorname{diag}\{\mathbf{W}(2)\} \mathbf{W}, \\
& =\sqrt{K M} \mathbf{W} \operatorname{diag}\left\{\mathbf{W}^{\mathrm{H}}(2)\right\} \mathbf{W}^{\mathrm{H}},
\end{aligned}
$$

where $\mathbf{W}(2)$ denotes the second column of $\mathbf{W}$. Since $\boldsymbol{\Pi}^{\mathrm{T}}$ is column wise up-shifting matrix, we have $\Pi^{\mathrm{T}}=\Pi^{\mathrm{H}}=\Pi^{-1}$. What is more, it is clear that $\Pi^{n}$ and $\Pi^{-n}$ represent $n$-times down shifting matrix and up shifting matrix respectably (Here we assume $n<K M$ for convenience). From (5), we have

$$
\begin{aligned}
\boldsymbol{\Pi}^{n} & =\sqrt{K M} \mathbf{W}^{\mathrm{H}} \operatorname{diag}\{\mathbf{W}(n+1)\} \mathbf{W} \\
\boldsymbol{\Pi}^{-n} & =\sqrt{K M} \mathbf{W}^{\mathrm{H}} \operatorname{diag}\left\{\mathbf{W}^{\mathrm{H}}(n+1)\right\} \mathbf{W} .
\end{aligned}
$$

Thus, from (2), (3), and (7), it is clear that

$$
\begin{array}{r}
\mathbf{W}^{2} \mathbf{U}=\mathbf{U} \overline{\mathbf{W}}^{2}, \\
\mathbf{W}^{2} \boldsymbol{\Pi}^{n}=\boldsymbol{\Pi}^{-n} \mathbf{W}^{2} .
\end{array}
$$

\section{Signal Model With IQI}

In this section, signal models for OFDMA and SC-FDMA in the presence of Tx IQI will be derived. First, we describe the IQ distorted received signal model for OFDMA with general subcarrier allocation. Then the impact of the interference due to the Tx IQIs at the conventional receiver is investigated. Furthermore, we specify the models to the systems with two major subcarrier allocations, namely interleaved and localized, and show the unfair IUIs depend on employed subcarrier allocations. After that, a system model for SC-FDMA including IFDMA will be shown. In the followings, we assume a system with $K$ users and the available $K M$ subcarriers are divided into $K$ subcarrier groups equally.

\section{A. OFDMA with General Subcarrier Allocation}

Fig. 1 depicts the block diagram of the OFDMA system of our interest. Let $\mathbf{s}_{k}=\left[s_{k}(0), s_{k}(1), \cdots, s_{k}(M-1)\right]^{\mathrm{T}}$ designate a block of $M$ data symbols transmitted by a user with index $k(k=1,2, \cdots, K)$. The data symbols may result from application of a modulation scheme like PSK or QAM to either forward error control coded or to uncoded data bits. In this paper, we discard the time index for convenience, since we will consider block by block transmissions and equalizations, and never refer to previous or subsequent blocks. The assignment of the data symbols $\mathbf{s}_{k}$ to the user specific set of $M$ subcarriers can be represented by $K M \times M$ mapping matrix $\mathbf{M}_{k}$ and a $K M$-point IDFT matrix $\mathbf{W}^{\mathrm{H}}$. The mapping matrix $\mathbf{M}_{k}$ has a unique element of one in each column and has properties of

$$
\mathbf{M}_{l}^{\mathrm{T}} \mathbf{M}_{k}=\left\{\begin{array}{ll}
\mathbf{0}_{M \times M} & (l \neq k) \\
\mathbf{I}_{M} & (l=k)
\end{array},\right.
$$

and

$$
\mathbf{M}_{l} \mathbf{M}_{k}^{\mathrm{T}}=\left\{\begin{array}{ll}
\mathbf{0}_{K M \times K M} & (l \neq k) \\
\operatorname{diag}\left\{\mathbf{m}_{k}\right\} & (k=l)
\end{array},\right.
$$

where the $K M \times 1$ vector $\mathbf{m}_{k}$ denotes the placement of the occupied subcarriers by the $k$ th user, whose elements consist of $M 1 \mathrm{~s}$ and $(K-1) M 0$ s. Using $\mathbf{M}_{k}$, the transmitted signal of the $k$ th user is given by

$$
\mathbf{x}_{k}=\mathbf{W}^{\mathrm{H}} \mathbf{M}_{k} \mathbf{s}_{k} .
$$

Before transmission, the baseband signal $\mathbf{x}_{k}$ is up-converted to the radio frequency signal by using a local oscillator 


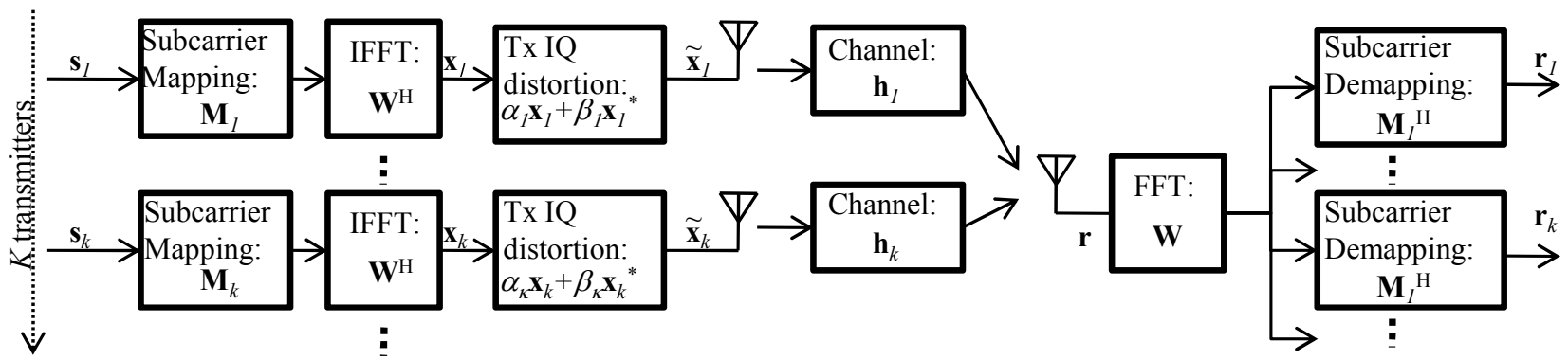

Fig. 1. The OFDMA system with Tx IQIs and the notation used in the derivations. Note that the insertion and removal of the cyclic prefix are not shown in this figure for ease of notations.

(LO) of the carrier frequency. Ideally, the LO outputs for the I and Q branches (representing the real part $\operatorname{Re}\left\{\mathbf{x}_{k}\right\}$ and imaginary parts $\operatorname{Im}\left\{\mathbf{x}_{k}\right\}$ ) should have equal amplitudes and phase difference of $\pi / 2$. However, in practice, the matching of I and Q signals is habitually imperfect and this leads to the amplitude and phase imbalance between the I and Q signals. Such impairments are known as Tx IQ imbalance or mismatch and this severely limits the performance of the receiver, especially if cheap components or architectures, e.g., direct conversion architecture [18], are employed. The IQ distorted signal of the $k$ th user can be modeled using the original signal $\mathbf{x}_{k}$ and its conjugate [6], [7] as

$$
\tilde{\mathbf{x}}_{k}=\alpha_{k} \mathbf{x}_{k}+\beta_{k} \mathbf{x}_{k}^{*}
$$

where two complex scalers $\alpha_{k}$ and $\beta_{k}$ are given by

$$
\begin{aligned}
& \alpha_{k}=\cos \theta_{k}+j \cdot \epsilon_{k} \sin \theta_{k}, \\
& \beta_{k}=\epsilon_{k} \cos \theta_{k}-j \cdot \sin \theta_{k},
\end{aligned}
$$

where $\theta_{k}$ and $\epsilon_{k}$ denote the phase and amplitude imbalances between the I and Q branches of the transmitted signal of the $k$ th user, respectively. When the matching of I and Q balances is ideal, i. e., $\theta_{k}=0$ and $\epsilon_{k}=0$, then $\alpha_{k}=1$ and $\beta_{k}=0$. The degree of the imbalance is evaluated by using the image rejection ratio (IRR), for the $k$ th user's transmitted signal, which is defined by

$$
\operatorname{IRR}_{k}=\frac{\mathrm{E}\left[\left\|\alpha_{k} \mathbf{x}_{k}\right\|^{2}\right]}{\mathrm{E}\left[\left\|\beta_{k} \mathbf{x}_{k}^{*}\right\|^{2}\right]}=\frac{\left|\alpha_{k}\right|^{2}}{\left|\beta_{k}\right|^{2}} .
$$

Let $h_{k}(t)(t=0,1, \cdots, L-1)$ denote a channel impulse response of the $k$ th user where $L-1$ is the order of the channel, and the $K M \times 1$ vector $\mathbf{h}_{k}$ is defined as $\mathbf{h}_{k}:=\left[h_{k}(0), h_{k}(1), \cdots, h_{k}(L-1), \mathbf{0}_{1 \times K M-L}\right]^{\mathrm{T}}$. Before the transmission over the channel $\mathbf{h}_{k}$, cyclic prefix $(\mathrm{CP})$ is appended to $\mathbf{x}_{k}$, which is removed at the receiver before the demodulation. When the time duration of $\mathrm{CP}$ is longer than that of the channel, it is well known that insertion of the $\mathrm{CP}$, transmission over the channel, and removal of the $\mathrm{CP}$ at the receiver can be described by an equivalent $K M \times K M$ circulant channel matrix $\mathbf{H}_{k}$, whose first column is given by $\mathbf{h}_{k}$. Since the circulant matrix can be diagonalized by DFT matrix, the received signal block after $\mathrm{CP}$ removal is described as,

$$
\begin{aligned}
\mathbf{r} & =\sum_{k=1}^{K} \mathbf{H}_{k} \tilde{\mathbf{x}}_{k}+\mathbf{n} \\
& =\mathbf{W}^{\mathrm{H}}\left(\sum_{k=1}^{K} \alpha_{k} \boldsymbol{\Lambda}_{k} \mathbf{M}_{k} \mathbf{s}_{k}+\beta_{k} \boldsymbol{\Lambda}_{k} \mathbf{W}^{2} \mathbf{M}_{k} \mathbf{s}_{k}^{*}\right)+\mathbf{n},
\end{aligned}
$$

where $\boldsymbol{\Lambda}_{k}:=\operatorname{diag}\left\{\mathbf{W} \mathbf{h}_{k}\right\}$, and the $K M \times 1$ vector $\mathbf{n}$ denotes additive noise. In addition, even if the length of CP is insufficient, we can reduce the ill effect of insufficient $\mathrm{CP}$ by using some advanced signal processing at the receiver, e.g., [15]. It should be mentioned that the IQ distortion is also introduced at frequency down-conversion at the receiver. In this paper, since we consider the uplink transmission, we assume that the IQI at the receiver, e.g., a base station, is negligibly small compared with that of the transmitters, e.g., mobile terminals, or has a priori compensated with some advanced digital signal processing techniques such as [8], [11], and we only deal with the Tx IQIs.

In addition, though we only resort to the frequency-flat IQI as in (14), the frequency-selective IQI [22] together with carrier frequency offset (CFO) is also notable analog impairments in wideband systems. We can find some joint compensation methods for IQI and CFO in [23] and [24]. In this paper, we mainly study the IUI problem due to Tx IQIs in OFDM based multiple access schemes and only resort to the frequency-flat case for simplicity.

Next, we describe the per-user received signal after multiuser detection. Here, we assume that the user separation is performed in the conventional manner, i.e., the received signal component on the set of subcarriers assigned to a user is extracted out by using the corresponding allocation matrix in discrete frequency domain. The received signal of the $l$ th user $(l=1,2, \cdots, K)$ after user separation is given by

$$
\begin{aligned}
\mathbf{r}_{l} & =\mathbf{M}_{l}^{\mathrm{T}} \mathbf{W r} \\
& =\alpha_{l} \boldsymbol{\Lambda}_{l l} \mathbf{s}_{l}+\left(\sum_{k=1}^{K} \beta_{k} \boldsymbol{\Lambda}_{l k} \mathbf{M}_{l}^{\mathrm{T}} \mathbf{W}^{2} \mathbf{M}_{k} \mathbf{s}_{k}^{*}\right)+\mathbf{n}_{l},
\end{aligned}
$$

where $\boldsymbol{\Lambda}_{l k}=\operatorname{diag}\left\{\mathbf{M}_{l}^{\mathrm{T}} \mathbf{W} \mathbf{h}_{k}\right\}$, and $\mathbf{n}_{l}=\mathbf{M}_{l}^{\mathrm{T}} \mathbf{W} \mathbf{n}$. The second term in the right-hand side of (19) represents the interference due to the Tx IQIs. Recalling (2) and $\mathbf{M}_{k}$ is the mapping matrix, $\mathbf{M}_{l} \mathbf{W}^{2} \mathbf{M}_{k}$ consist of 0 or 1 elements and hence the appearance of the interference largely depend on the 
employed subcarrier mappings. In order to clarify the effect of the interference on the performance of OFDMA receivers, we derive the per-subcarrier SIRs for each user. By assuming,

$$
\mathrm{E}\left[\mathbf{s}_{k} \mathbf{s}_{l}^{\mathrm{H}}\right]=\left\{\begin{array}{ll}
\sigma_{s}^{2} \mathbf{I}_{M} & (k=l) \\
\mathbf{0}_{M \times M} & (\text { otherwise })
\end{array},\right.
$$

we have

$$
\begin{aligned}
& \mathbf{R}_{l}^{(\mathrm{s})}=\mathrm{E}\left[\mathbf{r}_{l}^{(\mathrm{s})}\left(\mathbf{r}_{l}^{(\mathrm{s})}\right)^{H}\right]=\left|\alpha_{l}\right|^{2} \sigma_{s}^{2} \boldsymbol{\Lambda}_{l l} \boldsymbol{\Lambda}_{l l}^{*} \\
& \mathbf{R}_{l}^{(\mathrm{i})}=\mathrm{E}\left[\mathbf{r}_{l}^{(\mathrm{i})}\left(\mathbf{r}_{l}^{(\mathrm{i})}\right)^{H}\right]=\sum_{k=1}^{K}\left|\beta_{k}\right|^{2} \sigma_{s}^{2} \boldsymbol{\Lambda}_{l k} \boldsymbol{\Lambda}_{l k}^{*} \operatorname{diag}\left\{\mathbf{M}_{l}^{\mathrm{T}} \mathbf{W}^{2} \mathbf{m}_{k}\right\} .
\end{aligned}
$$

where $\mathbf{r}_{l}^{(\mathrm{s})}=\alpha_{l} \boldsymbol{\Lambda}_{l l} \mathbf{s}_{l}$ and $\mathbf{r}_{l}^{(\mathrm{i})}=\sum_{k=1}^{K} \beta_{k} \boldsymbol{\Lambda}_{l k} \mathbf{M}_{l}^{\mathrm{T}} \mathbf{W}^{2} \mathbf{M}_{k} \mathbf{s}_{k}^{*}$. Therefore, the SIR of the $m$ th subcarrier $(m=1, \cdots, M)$ in the $l$ th user's received signal is given by

$$
\begin{aligned}
& \operatorname{SIR}_{l}(m)=\frac{\mathbf{R}_{l}^{(\mathrm{s})}(m, m)}{\mathbf{R}_{l}^{(\mathrm{i})}(m, m)} \\
& =\frac{\left|\alpha_{l}\right|^{2}}{\left|\beta_{l}\right|^{2} \mathbf{m}_{l l}^{\prime}(m)+\sum_{k=1}^{K}(k \neq l)\left|\beta_{k}\right|^{2} \cdot \frac{\left|\mathbf{\Lambda}_{l k}(m)\right|^{2}}{\left|\mathbf{\Lambda}_{l l}(m)\right|^{2}} \cdot \mathbf{m}_{l k}^{\prime}(m)},
\end{aligned}
$$

where we set $\mathbf{m}_{l k}^{\prime}=\operatorname{diag}\left\{\mathbf{M}_{l}^{\mathrm{T}} \mathbf{W}^{2} \mathbf{m}_{k}\right\}$ and $\boldsymbol{\Lambda}_{l k}(m)$ denotes the $m$ th diagonal element of $\boldsymbol{\Lambda}_{l k}$. In the denominator of (23), the first term corresponds to the interference due to the $l$ th user's own Tx IQI. Meanwhile, the second term comes from the interference caused by other users' IQIs, namely the IUI. For comparison, we also consider the OFDM system in the presence of the Tx IQI, or equivalently $K=1$ and the mapping matrix $\mathbf{M}_{1}=\mathbf{I}_{M}$ in our formulations. In this case, the received signal is given by

$$
\mathbf{r}_{1}=\alpha_{1} \boldsymbol{\Lambda}_{11} \mathbf{s}_{1}+\beta_{1} \boldsymbol{\Lambda}_{11} \mathbf{W}^{2} \mathbf{s}_{1}^{*}+\mathbf{n}_{1} .
$$

Thus, the per-subcarrier received SIR results in

$$
\operatorname{SIR}_{1}(m)=\frac{\left|\alpha_{1}\right|^{2}}{\left|\beta_{1}\right|^{2}} .
$$

Obviously, there is no IUI problem in OFDM system and the $\operatorname{SIR}_{1}(m)$ is the same as the $I R R_{l}$. Therefore high IRR level at the transmitter's analog front-end directly means low interference power due to the Tx IQI at the receiver. Meanwhile, in the OFDMA system, the per-user received signal suffer from the IUI depending on the employed subcarrier allocation and the resulting $\operatorname{SIR}_{l}(m)$ is far from the $\operatorname{IRR}_{l}$. Furthermore, since the impact of such IUI is proportional to the corresponding channel gains $\frac{\left|\boldsymbol{\Lambda}_{l k}(m)\right|^{2}}{\left|\boldsymbol{\Lambda}_{l l}(m)\right|^{2}}$ as in (23), $\operatorname{SIR}_{l}(m)$ possibly comes down to the serious level due to the IUI in selective fading environments. This suggests essential need of Tx IQI compensation in OFDMA systems. Heretofore, the Tx IQI compensation is considered at the transmitter to simply improve the quality of IQ modulation, or at the receiver to avoid the additional complexity at the transmitter [9]. Here, in OFDMA systems, we can newly say that the Tx IQI compensation at the receiver is much more effective than at the transmitters, because achieving the high IRR level at the transmitter is not necessary meaning the low SIR at the

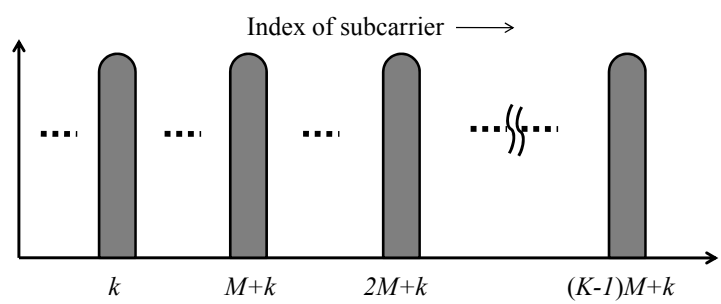

Fig. 2. Subcarrier allocation of $k$ th user in the interleaved OFDMA

receiver. From this perspective, we will propose the simple Tx IQI compensation methods for OFDMA receivers in Sec. III. Before that, for further analysis of the impact of the IUI, we specify the signal models for OFDMA systems with two major subcarrier allocations, i.e., interleaved and localized allocations and also derive the received SIR for each subcarrier allocation.

\section{B. Interleaved OFDMA}

Interleaved OFDMA, also known as distributed OFDMA has the user dependent comb shape subcarrier allocation depicted in Fig. 2. Such mapping matrix $\mathbf{M}_{k}$ for the $k$ th user is given by

$$
\mathbf{M}_{k}=\boldsymbol{\Pi}^{k-1} \mathbf{U} .
$$

Thus from (19), we have the $l$ th user received signal for the interleaved OFDMA as

$$
\begin{gathered}
\mathbf{r}_{l}=\alpha_{l} \boldsymbol{\Lambda}_{l l} \mathbf{s}_{l}+\left(\sum_{k=1}^{K} \beta_{k} \boldsymbol{\Lambda}_{l k} \mathbf{U}^{\mathrm{T}} \boldsymbol{\Pi}^{-l+1} \mathbf{W}^{2} \boldsymbol{\Pi}^{k-1} \mathbf{U} \mathbf{s}_{k}^{*}\right)+\mathbf{n}_{l} \\
=\alpha_{l} \boldsymbol{\Lambda}_{l l} \mathbf{s}_{l}+\left(\sum_{k=1}^{K} \beta_{k} \boldsymbol{\Lambda}_{l k} \mathbf{U}^{\mathrm{T}} \boldsymbol{\Pi}^{-l-k+2} \mathbf{U} \overline{\mathbf{W}}^{2} \mathbf{s}_{k}^{*}\right)+\mathbf{n}_{l}(27)
\end{gathered}
$$

From (3) and (7),

$$
\mathbf{U}^{\mathrm{T}} \boldsymbol{\Pi}^{-n} \mathbf{U}=\sqrt{\frac{M}{K}} \overline{\mathbf{W}}^{\mathrm{H}} \operatorname{diag}\{\mathbf{p}\} \overline{\mathbf{W}}, \quad(n=0, \cdots, 2 K-2)
$$

where the $m$ th element of the vector $\mathbf{p}$ is given by

$$
\begin{aligned}
& \mathbf{p}(m)=\frac{1}{\sqrt{K M}} \sum_{k=1}^{K} e^{j \cdot 2 \pi \frac{(m-1+k M) n}{K M}} \\
& = \begin{cases}\sqrt{K} \overline{\mathbf{W}}^{\mathrm{H}}(m, 1) & (n=0) \\
\sqrt{K} \overline{\mathbf{W}}^{\mathrm{H}}(m, 2) & (n=K) \\
\mathbf{0}_{M \times 1} & \text { (otherwise) }\end{cases}
\end{aligned}
$$

Therefore

$$
\mathbf{U}^{\mathrm{T}} \boldsymbol{\Pi}^{-n} \mathbf{U}=\left\{\begin{array}{ll}
\mathbf{I}_{M} & (n=0) \\
\overline{\boldsymbol{\Pi}}^{-1} & (n=K) \\
\mathbf{0}_{M \times M} & \text { (otherwise) }
\end{array},\right.
$$

where $\overline{\boldsymbol{\Pi}}^{-i}:=\sqrt{M} \overline{\mathbf{W}}^{\mathrm{H}} \operatorname{diag}\left\{\overline{\mathbf{W}}^{\mathrm{H}}(i+1)\right\} \overline{\mathbf{W}}$ denotes the $i$ times up shifting matrix of $M \times M$. As a result, we can further simplify the per-user received signal as

$$
\mathbf{r}_{l}=\alpha_{l} \boldsymbol{\Lambda}_{l l} \mathbf{s}_{l}+\beta_{f(l)} \boldsymbol{\Lambda}_{l f(l)} \overline{\mathbf{\Pi}}^{-g(l)} \overline{\mathbf{W}}^{2} \mathbf{s}_{f(l)}^{*}+\mathbf{n}_{l},
$$




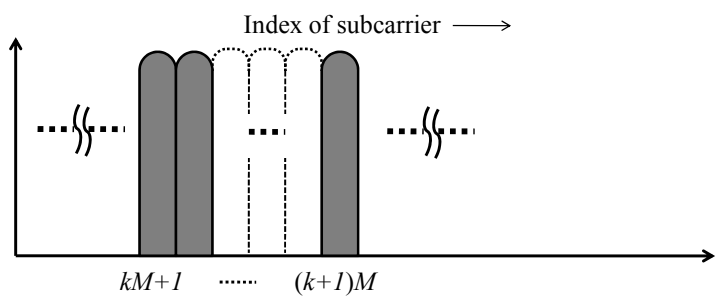

Fig. 3. Subcarrier allocation of $k$ th user in the localized OFDMA

where $f(l)$ and $g(l)$ are given by

$$
\begin{aligned}
& f(l)=\left\{\begin{array}{ll}
1 & (l=1) \\
K-l+2 & (\text { otherwise })
\end{array},\right. \\
& g(l)= \begin{cases}0 & (l=1) \\
1 & (\text { otherwise })\end{cases}
\end{aligned}
$$

For example, when $K=4$, the relation ship between $l$ and $f(l)$ is given as follows,

$$
\begin{array}{c|cccc}
l & 1 & 2 & 3 & 4 \\
\hline f(l) & 1 & 4 & 3 & 2
\end{array} .
$$

Under the same assumption in (20), the per-subcarrier received SIR of the $l$ th user is given by

$$
\begin{aligned}
\operatorname{SIR}_{l}(m) & =\frac{\mathrm{E}\left[\left|\alpha_{l} \boldsymbol{\Lambda}_{l l}(m) \mathbf{s}_{l}(m)\right|^{2}\right]}{\mathrm{E}\left[\left|\beta_{f(l)} \boldsymbol{\Lambda}_{l f(l)}(m)\left(\overline{\boldsymbol{\Pi}}^{-g(l)} \overline{\mathbf{W}}^{2} \mathbf{s}_{f(l)}^{*}\right)(m)\right|^{2}\right]} \\
& =\frac{\left|\alpha_{l} \boldsymbol{\Lambda}_{l l}(m)\right|^{2}}{\left|\beta_{f(l)} \boldsymbol{\Lambda}_{l f(l)}(m)\right|^{2}}
\end{aligned}
$$

where $m=1, \cdots, M$. Interestingly, in the interleaved OFDMA system, the $l$ th user's received signal interfered only by the $f(l)$ th user's image component. In particular, when $l \neq f(l)$, the interference can be considered as an IUI and, with respect to user fairness, such IUI is quite undesirable since the impairment in IQ modulation of a user terminal make trouble not on its own performance but on the other's. What is more, from (34), the power of such IUI is proportional to the ratio of the channel gains and the resulting $\operatorname{SIR}_{l}(m)$ ( $m=1,2, \cdots, M)$ is far from $\mathrm{IRR}_{l}$. On the other hand, the 1 st and the $(K / 2+1)$ th users successfully avoid the IUI and the resulting SIR is equivalent to the IRR. We evaluate this unique phenomenon later in our computer simulations.

\section{Localized OFDMA}

The localized OFDMA has block-wise subcarrier allocation as in Fig. 3, and the mapping matrix for the $k$ th user is given by

$$
\mathbf{M}_{k}=\mathbf{\Pi}^{(k-1) M} \mathbf{V},
$$

where $K M \times M$ matrix $\mathbf{V}$ is defined as

$$
\mathbf{V}=\left[\begin{array}{c}
\mathbf{I}_{M} \\
\mathbf{0}_{(K-1) M \times M}
\end{array}\right] \text {. }
$$

Therefore, the per-user received signal is represented by

$$
\begin{aligned}
& \mathbf{r}_{l}= \\
& \alpha_{l} \boldsymbol{\Lambda}_{l l} \mathbf{s}_{l}+\left(\sum_{k=1}^{K} \beta_{k} \boldsymbol{\Lambda}_{l k} \mathbf{V}^{\mathrm{T}} \mathbf{\Pi}^{-(l-1) M} \mathbf{W}^{2} \mathbf{\Pi}^{(k-1) M} \mathbf{V} \mathbf{s}_{k}^{*}\right)+\mathbf{n}_{l} \\
& =\alpha_{l} \boldsymbol{\Lambda}_{l l} \mathbf{s}_{l}+\left(\sum_{k=1}^{K} \beta_{k} \boldsymbol{\Lambda}_{l k} \mathbf{V}^{\mathrm{T}} \boldsymbol{\Pi}^{-(k+l-2) M} \mathbf{W}^{2} \mathbf{V} \mathbf{s}_{k}^{*}\right)+\mathbf{n}_{l} .
\end{aligned}
$$

Here

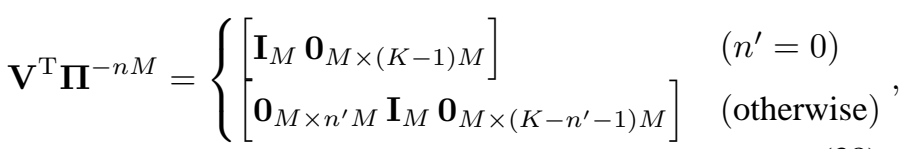

where $n^{\prime}:=n \bmod K$, and $\mathbf{W}^{2} \mathbf{V}$ denotes $K M \times M$ matrix consist of the first $M$ columns of $\mathbf{W}^{2}$, i.e.,

$$
\mathbf{W}^{2} \mathbf{V}=\left[\begin{array}{c}
\mathbf{J} \\
\mathbf{0}_{(K-2) M \times M} \\
\overline{\mathbf{W}}^{2}-\mathbf{J}
\end{array}\right]
$$

where the (m, n) element of $M \times M$ matrix $\mathbf{J}$ is defined as

$$
\mathbf{J}(m, n)=\left\{\begin{array}{ll}
1 & (m=n=1) \\
0 & \text { (otherwise) }
\end{array} .\right.
$$

Therefore, we can simplify (37) as

$\mathbf{r}_{l}=\alpha_{l} \boldsymbol{\Lambda}_{l l} \mathbf{s}_{l}+\beta_{f(l)} \boldsymbol{\Lambda}_{l f(l)} \mathbf{J}_{f(l)}^{*}+\beta_{e(l)} \boldsymbol{\Lambda}_{l e(l)}\left(\overline{\mathbf{W}}^{2}-\mathbf{J}\right) \mathbf{s}_{e(l)}^{*}+\mathbf{n}_{l},$,

where

$$
e(l)=K-l+1 .
$$

For example, when $K=4$,

$$
\begin{array}{c|cccc}
l & 1 & 2 & 3 & 4 \\
\hline e(l) & 4 & 3 & 2 & 1
\end{array}
$$

The resulting per-subcarrier SIR of the $l$ th user is given by

$$
\operatorname{SIR}_{l}(m)= \begin{cases}\frac{\left|\alpha_{l} \boldsymbol{\Lambda}_{l l}(m)\right|^{2}}{\left.\left|\beta_{f(l)} \boldsymbol{\Lambda}_{l}(l)\right|(m)\right|^{2}} & (m=1) \\ \frac{\left|\alpha_{l} \boldsymbol{\Lambda}_{l l}(m)\right|^{2}}{\left|\beta_{e(l)} \boldsymbol{\Lambda}_{l e(l)}(m)\right|^{2}} & (m>1)\end{cases}
$$

where $m=1, \cdots, M$. In the localized OFDMA, the interference on the $l$ th user received signal consist of the IUIs from $f(l)$ and $e(l)$ th user's image components. Actually, from (44), the IUI from the $e(l)$ th user is the main source of the performance degradation when $M$ is sufficiently large.

\section{SC-FDMA}

A system model for SC-FDMA is easily derived in the context of DFT-precoded OFDMA [3], i.e., to replace $\mathbf{s}_{k}$ by $\overline{\mathbf{W}} \mathbf{s}_{k}$. Thus from (19), the $k$ th user's transmitted signal is given by

$$
\mathbf{x}_{k}=\mathbf{W}^{\mathrm{H}} \mathbf{M}_{k} \overline{\mathbf{W}} \mathbf{s}_{k} .
$$


From (18), the received signal is represented by

$$
\mathbf{r}=\mathbf{W}^{\mathrm{H}}\left(\sum_{k=1}^{K} \alpha_{k} \boldsymbol{\Lambda}_{k} \mathbf{M}_{k} \overline{\mathbf{W}} \mathbf{s}_{k}+\beta_{k} \boldsymbol{\Lambda}_{k} \mathbf{W}^{2} \mathbf{M}_{k} \overline{\mathbf{W}}^{\mathrm{H}} \mathbf{s}_{k}^{*}\right)+\mathbf{n} .
$$

Correspondingly, the received signal component of the $l$ th user is represented as

$$
\begin{aligned}
\mathbf{r}_{l}= & \overline{\mathbf{W}}^{\mathrm{H}} \mathbf{M}_{l}^{\mathrm{T}} \mathbf{W r}=\alpha_{l} \overline{\mathbf{W}}^{\mathrm{H}} \boldsymbol{\Lambda}_{l l} \overline{\mathbf{W}} \mathbf{s}_{l} \\
& +\left(\sum_{k=1}^{K} \beta_{k} \overline{\mathbf{W}}^{\mathrm{H}} \boldsymbol{\Lambda}_{l k} \mathbf{M}_{l}^{\mathrm{T}} \mathbf{W}^{2} \mathbf{M}_{k} \overline{\mathbf{W}} \mathbf{s}_{k}^{*}\right)+\mathbf{n}_{l},
\end{aligned}
$$

where the noise vector $\mathbf{n}_{l}=\overline{\mathbf{W}}^{\mathrm{H}} \mathbf{M}_{l}^{\mathrm{T}} \mathbf{W} \mathbf{n}$.

Two major choices of $\mathbf{M}_{k}$ are localized and interleaved allocation. It should be mentioned that, when the interleaved subcarrier allocation is employed, the subcarrier assignment for each user can be further simplified, i.e.,

$$
\begin{aligned}
\mathbf{x}_{k} & =\mathbf{W}^{\mathrm{H}} \boldsymbol{\Pi}^{k-1} \mathbf{U} \overline{\mathbf{W}} \mathbf{s}_{k} \\
& =\sqrt{\frac{M}{K}} \operatorname{diag}\left\{\mathbf{W}^{\mathrm{H}}(k)\right\}\left[\begin{array}{c}
\mathbf{I}_{M} \\
\vdots \\
\mathbf{I}_{M}
\end{array}\right] \mathbf{s}_{k} .
\end{aligned}
$$

This shows that the transmitted signal is easily designed based on repetition and subsequent user dependent frequency shift of a modulated signal. In such case, the interleaved SC-FDMA is known as IFDMA [4]. IFDMA is one promising alternative to OFDMA due to the low complexity of user separation as well as the low PAPR of the transmitted signal, especially for the mobile uplink.

In regard to the per-subcarrier received SIR, since the DFT matrix $\mathbf{W}$ is a unitary, thus we have $\mathrm{E}\left[\left(\mathbf{W} \mathbf{s}_{k}\right)(m)\left(\mathbf{W} \mathbf{s}_{k}\right)^{\mathrm{H}}(m)\right]=\mathrm{E}\left[\mathbf{s}_{k}(m) \mathbf{s}_{k}^{\mathrm{H}}(m)\right]$ under the same assumption in (20) and hence the per-subcarrier SIRs for the SC-FDMA results in the same as those of OFDMA.

\section{WIDELY LINEAR EQUALIZATION AND USER Detection Method In THE PREsence of TX IQI}

Here, we consider the equalization and the multiple user detection problem at the OFDMA receiver in the presence of Tx IQIs.

From (18), the received signal $\mathbf{r}$ is not the linear function of $\mathbf{s}_{k}(k=1, \cdots, K)$ due to the Tx IQI, however, the regression is linear both in $\mathbf{s}_{k}$ and $\mathbf{s}_{k}^{*}$. Such system is known as widely linear (WL) or conjugate linear system. The WL filtering approach [17], which jointly elaborate the received signal and its conjugate, can be efficiently applied to the equalization and the user detection problems in such WL systems. In this section, first, the WL equalization and user detection method for the OFDMA receiver with general subcarrier allocation will be proposed. One attractive feature of the conventional OFDMA receiver is the efficient user separation and channel equalization in discrete frequency domain [2], [19]. The proposed receiver is also implemented in discrete frequency domain, and can jointly compensate the Tx IQI and the channel. Moreover, we propose a simple pairwise equalizer for the OFDMA receiver with interleaved subcarrier allocation. In the previous section, we have shown the IUI due to the Tx IQIs appears only between certain pairs of users in the OFDMA. Making use of the fact, we consider a pairwise equalization scheme for user-separated signals in the interleaved OFDMA receiver, which can further reduce the complexity. Both for the general and interleaved OFDMA systems, the WL receiver based on ZF and MMSE criteria will be derived where we assume all the channel state information and the Tx IQI parameters are a priori known to the receiver by using some sophisticated signal processing techniques such as [9] or [11]. It should be mentioned that, in this section, we mainly deal with OFDMA. However, the proposed methods can be easily applied to the SC-FDMA in the context of the DFT-precoded OFDMA as in Sec 2. D.

\section{A. The WL receiver for OFDMA with general subcarrier allocation}

Here, we propose the WL receiver for the OFDMA system with general subcarrier allocation. From (18),

$$
\mathbf{r}=\mathbf{W}^{\mathrm{H}} \operatorname{diag}\{\boldsymbol{\gamma}\} \mathbf{s}+\mathbf{W}^{\mathrm{H}} \operatorname{diag}\{\boldsymbol{\delta}\} \mathbf{W}^{2} \mathbf{s}^{*}+\mathbf{n},
$$

where

$\mathbf{s}=\sum_{k=1}^{K} \mathbf{M}_{k} \mathbf{s}_{k}, \quad \gamma=\sum_{k=1}^{K} \alpha_{k} \boldsymbol{\Lambda}_{k} \mathbf{m}_{k}, \quad \boldsymbol{\delta}=\sum_{k=1}^{K} \beta_{k} \boldsymbol{\Lambda}_{k} \mathbf{W}^{2} \mathbf{m}_{k}$

Furthermore, by stacking $\mathbf{r}$ and $\mathbf{r}^{*}$, we have

$$
\left[\begin{array}{c}
\mathbf{r} \\
\mathbf{r}^{*}
\end{array}\right]=\left[\begin{array}{cc}
\mathbf{W}^{\mathrm{H}} \operatorname{diag}\{\boldsymbol{\gamma}\} & \mathbf{W}^{\mathrm{H}} \operatorname{diag}\{\boldsymbol{\delta}\} \mathbf{W}^{2} \\
\mathbf{W} \operatorname{diag}\left\{\boldsymbol{\delta}^{*}\right\} \mathbf{W}^{2} & \mathbf{W} \operatorname{diag}\left\{\boldsymbol{\gamma}^{*}\right\}
\end{array}\right]\left[\begin{array}{c}
\mathbf{s} \\
\mathbf{s}^{*}
\end{array}\right]+\left[\begin{array}{c}
\mathbf{n} \\
\mathbf{n}^{*}
\end{array}\right] .
$$

Clearly, we can separate each user's transmitted signal from $\mathbf{s}$ by using (11), e.g., $\mathbf{s}_{k}=\mathbf{M}_{k}^{\mathrm{T}} \mathbf{s}$. Therefore, in the followings, we discuss the estimation of $\mathrm{s}$ based on the ZF and MMSE criterion in the system (51).

1) $Z F$ equalizer: In the absence of the noise, the WL $Z F$ condition is given by

$$
\mathbf{s}=\mathbf{G}_{\mathrm{ZF}}\left[\begin{array}{c}
\mathbf{r} \\
\mathbf{r}^{*}
\end{array}\right]
$$

where $K M \times 2 K M$ matrix $\mathbf{G}_{\mathrm{ZF}}$ represents the $\mathrm{WL}$ equalizer. From (51), we can derive $\mathbf{G}_{\mathrm{ZF}}$ as

$$
\begin{aligned}
& \mathbf{G}_{\mathrm{ZF}}=
\end{aligned}
$$

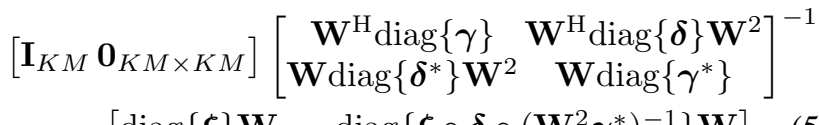

$$
\begin{aligned}
& =\left[\operatorname{diag}\{\boldsymbol{\xi}\} \mathbf{W}-\operatorname{diag}\left\{\boldsymbol{\xi} \circ \boldsymbol{\delta} \circ\left(\mathbf{W}^{2} \boldsymbol{\gamma}^{*}\right)^{-1}\right\} \mathbf{W}\right],
\end{aligned}
$$

where

$$
\boldsymbol{\xi}=\mathbf{W}^{2} \boldsymbol{\gamma}^{*} \circ\left(\boldsymbol{\gamma} \circ \mathbf{W}^{2} \boldsymbol{\gamma}^{*}-\boldsymbol{\delta} \circ \mathbf{W}^{2} \boldsymbol{\delta}^{*}\right)^{-1} .
$$

In the above equations, o denotes the element wise multiplication of vectors, i.e., $\mathbf{x} \circ \mathbf{y}=\operatorname{diag}\{\mathbf{x}\} \mathbf{y}$, and each element of vector $\mathbf{x}^{-1}$ is given by the reciprocal of the corresponding element of $\mathbf{x}$, i.e., $\mathbf{x}^{-1}(m)=1 / \mathbf{x}(m)$. Eq. (53) shows that, when $K M$ is power of 2 , the proposed WL ZF equalizer can be efficiently implemented by using FFT. In fact, it needs 
twice the computational effort as the one-tap FDE used in conventional OFDMA systems [1], [19].

It should be mentioned that when we employ noncircular modulation schemes including real modulation format such as BPSK, $m$-ASK or OQAM, we can find a certain relationship between $\mathbf{s}$ and $\mathbf{s}^{*}$ and this enables us to gain the additional degrees of freedom in choosing WL equalizer [21]. The exploitation of such noncircular property of the transmitted signal is one notable advantage of WL approach over conventional linear approach. However, in practical OFDMA and SCFDMA systems, circular modulation schemes such as QPSK and QAM scheme where $\mathbf{s}$ and $\mathbf{s}^{*}$ have the orthogonality, i.e., $\mathbf{s}^{*}$ cannot be obtained from $\mathbf{s}$ by a simple linear relation, are mainly employed. Therefore, in this paper, we only deal with the circular modulation schemes and don't resort to exploiting non-circularity of the signal modulation.

In addition, the proposed WL ZF receiver is closely related to the two per-tone equalizer in [23]. In [23], the compensation method for the Tx IQ distortion together with the Rx IQI and the carrier frequency offset at the OFDM and OFDMA receivers has been proposed. The structure of the proposed $\mathrm{ZF}$ WL receiver results in the same as that of their two per-tone equalizer. In this paper, we explicitly derive the tap weights of the receiver. Furthermore, we will analytically show the optimal WL receiver in terms of the MMSE criterion is also realized by the same structure as the $\mathrm{ZF}$ receiver.

2) MMSE equalizer: As in [17], the best WL equalizer in terms of minimum error covariance, i. e.,

$$
\mathbf{G}_{\mathrm{MMSE}}=\arg \min _{\mathbf{G}} \mathrm{E}\left[\left\|\left[\begin{array}{c}
\mathbf{s} \\
\mathbf{s}^{*}
\end{array}\right]-\mathbf{G}\left[\begin{array}{c}
\mathbf{r} \\
\mathbf{r}^{*}
\end{array}\right]\right\|^{2}\right]
$$

is obtained as

$$
\begin{aligned}
& \mathbf{G}_{\mathrm{MMSE}}= \\
& {\left[\begin{array}{ll}
\left(\mathbf{R}_{s r}-\mathbf{C}_{s r} \mathbf{R}_{r r}^{*-1} \mathbf{C}_{r r}^{*}\right) \mathbf{D}_{r r}^{-1} & \left.\left(\mathbf{C}_{s r}-\mathbf{R}_{s r} \mathbf{R}_{r r}^{-1} \mathbf{C}_{r r}\right) \mathbf{D}_{r r}^{*-1}\right],
\end{array}\right.}
\end{aligned}
$$

where the correlation matrix $\mathbf{R}_{x y}=\mathrm{E}\left[\mathrm{xy}^{\mathrm{H}}\right]$ and the complementary correlation matrix $\mathbf{C}_{x y}=\mathrm{E}\left[\mathbf{x y} \mathbf{T}^{\mathrm{T}}\right]$ and $\mathbf{D}_{r r}=$ $\mathbf{R}_{r r}-\mathbf{C}_{r r} \mathbf{R}_{r r}^{*-1} \mathbf{C}_{r r}^{*}$. Here we consider the system with circular modulation schemes and eventually assume $\mathbf{R}_{s s}=$ $\sigma_{s}^{2} \mathbf{I}_{K M}$ and $\mathbf{C}_{s s}=\mathbf{0}_{K M \times K M}$. Also by assuming $\mathbf{n}$ is a zero-mean white circular complex Gaussian noise, $\mathbf{n} \sim$ $\mathcal{N}_{c}\left(\mathbf{0}_{K M \times 1}, \sigma_{n}^{2} \mathbf{I}_{K M}\right)$, we have

$$
\begin{aligned}
& \mathbf{R}_{s r}=\sigma_{s}^{2} \operatorname{diag}\left\{\boldsymbol{\gamma}^{*}\right\} \mathbf{W}, \\
& \mathbf{C}_{s r}=\sigma_{s}^{2} \operatorname{diag}\left\{\mathbf{W}^{2} \boldsymbol{\delta}\right\} \mathbf{W}, \\
& \mathbf{R}_{r r}=\sigma_{s}^{2} \mathbf{W}^{\mathrm{H}} \operatorname{diag}\left\{\langle\boldsymbol{\gamma}\rangle+\langle\boldsymbol{\delta}\rangle+\frac{\sigma_{n}^{2}}{\sigma_{s}^{2}} \mathbf{1}\right\} \mathbf{W}, \\
& \mathbf{C}_{r r}=\sigma_{s}^{2} \mathbf{W}^{\mathrm{H}} \operatorname{diag}\left\{\boldsymbol{\gamma} \circ \mathbf{W}^{2} \boldsymbol{\delta}+\boldsymbol{\delta} \circ \mathbf{W}^{2} \boldsymbol{\gamma}\right\} \mathbf{W},
\end{aligned}
$$

where $\langle\mathbf{x}\rangle=\mathbf{x} \circ \mathbf{x}^{*}$ and $\mathbf{1}$ denotes a $K M \times 1$ all one vector. Consequently, the WL MMSE equalizer is given by

$$
\mathbf{G}_{\text {MMSE }}=[\operatorname{diag}\{\mathbf{a}\} \mathbf{W} \quad \operatorname{diag}\{\mathbf{b}\} \mathbf{W}],
$$

where

$$
\begin{aligned}
\mathbf{a} & =\left\{\mathbf{W}^{2} \boldsymbol{\gamma}^{*} \circ\left(\boldsymbol{\gamma}^{*} \circ \mathbf{W}^{2} \boldsymbol{\gamma}-\boldsymbol{\delta}^{*} \circ \mathbf{W}^{2} \boldsymbol{\delta}\right)+\frac{\sigma_{n}^{2}}{\sigma_{s}^{2}} \boldsymbol{\gamma}^{*}\right\} \circ \boldsymbol{\nu}^{-1}, \\
\mathbf{b} & =\left\{-\boldsymbol{\delta} \circ\left(\boldsymbol{\gamma}^{*} \circ \mathbf{W}^{2} \boldsymbol{\gamma}-\boldsymbol{\delta}^{*} \circ \mathbf{W}^{2} \boldsymbol{\delta}\right)+\frac{\sigma_{n}^{2}}{\sigma_{s}^{2}} \mathbf{W}^{2} \boldsymbol{\delta}\right\} \circ \boldsymbol{\nu}^{-1} \\
\boldsymbol{\nu} & =\frac{\sigma_{n}^{2}}{\sigma_{s}^{2}}\left(\langle\boldsymbol{\gamma}\rangle+\langle\boldsymbol{\delta}\rangle+\mathbf{W}^{2}\langle\boldsymbol{\gamma}\rangle+\mathbf{W}^{2}\langle\boldsymbol{\delta}\rangle\right) \\
& +\left\langle\boldsymbol{\gamma} \circ \mathbf{W}^{2} \boldsymbol{\gamma}^{*}-\boldsymbol{\delta} \circ \mathbf{W}^{2} \boldsymbol{\delta}^{*}\right\rangle+\frac{\sigma_{n}^{4}}{\sigma_{s}^{4}} .
\end{aligned}
$$

As the proposed WL ZF equalizer, the proposed MMSE equalizer is also efficiently implemented in discrete frequency domain.

\section{B. The proposed pairwise equalizer for the interleaved OFDMA system}

Next, we consider the channel equalization in the interleaved OFDMA receiver with the conventional user separation. As in Sec. 2.2, when we employ the interleaved subcarrier allocation, the $l$ th user's received signal after the user separation only interfered with the $f(l)$ th user's image component. Taking the fact into consideration, we propose the pairwise equalizer for the interleaved OFDMA based on ZF and MMSE criteria. From (30), we have the pairwise received signal:

$$
\left[\begin{array}{c}
\mathbf{r}_{l} \\
\mathbf{r}_{f(l)}^{*}
\end{array}\right]=\left[\begin{array}{cr}
\operatorname{diag}\left\{\boldsymbol{\gamma}_{l}\right\} & \operatorname{diag}\left\{\boldsymbol{\delta}_{l}\right\} \boldsymbol{\Phi}_{l} \\
\operatorname{diag}\left\{\boldsymbol{\delta}_{f(l)}^{*}\right\} \boldsymbol{\Phi}_{l} \operatorname{diag}\left\{\boldsymbol{\gamma}_{f(l)}^{*}\right\}
\end{array}\right]\left[\begin{array}{c}
\mathbf{s}_{l} \\
\mathbf{s}_{f(l)}^{*}
\end{array}\right]+\left[\begin{array}{c}
\mathbf{n}_{l} \\
\mathbf{n}_{f(l)}^{*}
\end{array}\right]
$$

where

$$
\begin{aligned}
\operatorname{diag}\left\{\boldsymbol{\gamma}_{l}\right\} & =\alpha_{l} \boldsymbol{\Lambda}_{l l}, \\
\operatorname{diag}\left\{\boldsymbol{\delta}_{l}\right\} & =\beta_{f(l)} \boldsymbol{\Lambda}_{l f(l)}, \\
\boldsymbol{\Phi}_{l} & =\boldsymbol{\Phi}_{g(l)}=\overline{\boldsymbol{\Pi}}^{-g(l)} \overline{\mathbf{W}}^{2}
\end{aligned}
$$

1) $Z F$ equalizer: In the absence of the noise, the $Z F$ criterion is given by

$$
\mathbf{Z}_{\mathrm{ZF}}\left[\begin{array}{c}
\mathbf{r}_{l} \\
\mathbf{r}_{f(l)}^{*}
\end{array}\right]=\left[\begin{array}{c}
\mathbf{s}_{l} \\
\mathbf{s}_{f(l)}^{*}
\end{array}\right]
$$

where the $2 M \times 2 M$ matrix $\mathbf{Z}_{\mathrm{ZF}}$ denotes the linear $\mathrm{ZF}$ equalizer. Thus we have

$$
\begin{aligned}
\mathbf{Z}_{\mathrm{ZF}} & =\left[\begin{array}{cc}
\operatorname{diag}\left\{\boldsymbol{\gamma}_{l}\right\} & \operatorname{diag}\left\{\boldsymbol{\delta}_{l}\right\} \boldsymbol{\Phi}_{l} \\
\operatorname{diag}\left\{\boldsymbol{\delta}_{f(l)}^{*}\right\} \boldsymbol{\Phi}_{l} \operatorname{diag}\left\{\boldsymbol{\gamma}_{f(l)}^{*}\right\}
\end{array}\right] \\
& =\left[\begin{array}{cc}
\operatorname{diag}\left\{\mathbf{c}_{l}\right\} & \operatorname{diag}\left\{\mathbf{d}_{l}\right\} \boldsymbol{\Phi}_{l} \\
\operatorname{diag}\left\{\mathbf{d}_{f(l)}^{*}\right\} \boldsymbol{\Phi}_{l} \operatorname{diag}\left\{\mathbf{c}_{f(l)}^{*}\right\}
\end{array}\right]
\end{aligned}
$$

where $M$ size vector $\mathbf{c}_{l}$ and $\mathbf{d}_{l}$ denotes equalizer weights and they are given by

$$
\begin{aligned}
& \mathbf{c}_{l}=\boldsymbol{\Phi}_{l} \gamma_{f(l)}^{*} \circ\left(\gamma_{l} \circ \boldsymbol{\Phi}_{l} \gamma_{f(l)}^{*}-\boldsymbol{\delta}_{l} \circ \boldsymbol{\Phi}_{l} \boldsymbol{\delta}_{f(l)}^{*}\right)^{-1}, \\
& \mathbf{d}_{l}=-\boldsymbol{\delta}_{l} \circ\left(\gamma_{l} \circ \boldsymbol{\Phi}_{l} \gamma_{f(l)}^{*}-\boldsymbol{\delta}_{l} \circ \boldsymbol{\Phi}_{l} \boldsymbol{\delta}_{f(l)}^{*}\right)^{-1} .
\end{aligned}
$$




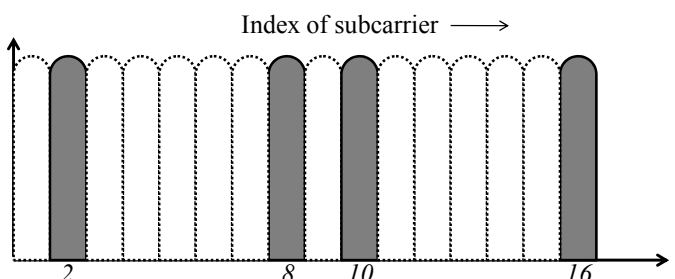

Fig. 4. Subcarrier allocation of the 2nd user in the proposed IUI free OFDMA where $K=4$ and $M=4$

2) MMSE equalizer: Under the same assumption in Sec. 3.1.B, we have

$$
\begin{aligned}
\mathbf{R}_{s_{l} r_{l}} & =\sigma_{s}^{2} \operatorname{diag}\left\{\boldsymbol{\gamma}_{l}^{*}\right\}, \\
\mathbf{C}_{s_{l} r_{f(l)}} & =\sigma_{s}^{2} \operatorname{diag}\left\{\boldsymbol{\Phi}_{l} \boldsymbol{\delta}_{f(l)}\right\} \boldsymbol{\Phi}_{l}, \\
\mathbf{R}_{r_{l} r_{l}} & =\sigma_{s}^{2} \operatorname{diag}\left\{\left\langle\gamma_{l}\right\rangle+\left\langle\boldsymbol{\delta}_{l}\right\rangle+\frac{\sigma_{n}^{2}}{\sigma_{s}^{2}} \mathbf{1}\right\}, \\
\mathbf{C}_{r_{l} r_{f(l)}} & =\sigma_{s}^{2} \operatorname{diag}\left\{\boldsymbol{\gamma}_{l} \circ \boldsymbol{\Phi}_{l} \boldsymbol{\delta}_{f(l)}+\boldsymbol{\delta}_{l} \circ \boldsymbol{\Phi}_{l} \boldsymbol{\gamma}_{f(l)}\right\} \boldsymbol{\Phi}_{l} .
\end{aligned}
$$

The optimum linear equalizer $\mathbf{Z}_{\text {MMSE }}$ based on MMSE criterion, i.e.,

$$
\mathbf{Z}_{\mathrm{MMSE}}=\arg \min _{\mathbf{R}} \mathrm{E}\left[\left\|\left[\begin{array}{c}
\mathbf{s}_{l} \\
\mathbf{s}_{f(l)}^{*}
\end{array}\right]-\mathbf{R}\left[\begin{array}{c}
\mathbf{r}_{l} \\
\mathbf{r}_{f(l)}^{*}
\end{array}\right]\right\|^{2}\right],
$$

can be derived as

$$
\begin{aligned}
\mathbf{Z}_{\text {MMSE }} & =\left[\begin{array}{cc}
\mathbf{R}_{s_{l} r_{l}} & \mathbf{C}_{s_{l} r_{f(l)}} \\
\mathbf{C}_{s_{f(l)} r_{l}}^{*} & \mathbf{R}_{s_{f(l)} r_{f(l)}}^{*}
\end{array}\right]\left[\begin{array}{cc}
\mathbf{R}_{r_{l} r_{l}} & \mathbf{C}_{r_{l} r_{f(l)}} \\
\mathbf{C}_{r_{f(l)} r_{l}}^{*} & \mathbf{R}_{r_{f(l)} r_{f(l)}}^{*}
\end{array}\right]^{-1} \\
& =\left[\begin{array}{cc}
\operatorname{diag}\left\{\mathbf{e}_{l}\right\} & \operatorname{diag}\left\{\mathbf{f}_{l}\right\} \boldsymbol{\Phi}_{l} \\
\operatorname{diag}\left\{\mathbf{f}_{f(l)}^{*}\right\} \boldsymbol{\Phi}_{l} \operatorname{diag}\left\{\mathbf{e}_{f(l)}^{*}\right\}
\end{array}\right]
\end{aligned}
$$

where

$$
\begin{aligned}
\mathbf{e}_{l} & =\left\{\boldsymbol{\Phi}_{l} \boldsymbol{\gamma}_{f(l)}^{*} \circ\left(\gamma_{l}^{*} \circ \boldsymbol{\Phi}_{l} \boldsymbol{\gamma}_{f(l)}-\boldsymbol{\delta}_{l}^{*} \circ \boldsymbol{\Phi}_{l} \boldsymbol{\delta}_{f(l)}\right)\right. \\
& \left.+\frac{\sigma_{n}^{2}}{\sigma_{s}^{2}} \boldsymbol{\gamma}_{l}^{*}\right\} \circ \boldsymbol{\nu}_{l}^{-1} \\
\mathbf{f}_{l} & =\left\{-\boldsymbol{\delta}_{l} \circ\left(\gamma_{l}^{*} \circ \boldsymbol{\Phi}_{l} \gamma_{f(l)}-\boldsymbol{\Phi}_{l} \boldsymbol{\delta}_{f(l)} \circ \boldsymbol{\delta}_{l}^{*}\right)\right. \\
& \left.+\frac{\sigma_{n}^{2}}{\sigma_{s}^{2}} \boldsymbol{\Phi}_{l} \boldsymbol{\delta}_{f(l)}\right\} \circ \boldsymbol{\nu}_{l}^{-1},
\end{aligned}
$$

and

$$
\begin{aligned}
\boldsymbol{\nu}_{l} & =\frac{\sigma_{n}^{2}}{\sigma_{s}^{2}}\left(\left\langle\gamma_{l}\right\rangle+\left\langle\boldsymbol{\delta}_{l}\right\rangle+\boldsymbol{\Phi}_{l}\left\langle\gamma_{f(l)}\right\rangle+\boldsymbol{\Phi}_{l}\left\langle\boldsymbol{\delta}_{f(l)}\right\rangle\right) \\
& +\left\langle\gamma_{l} \circ \boldsymbol{\Phi}_{l} \gamma_{f(l)}^{*}-\boldsymbol{\delta}_{l} \circ \boldsymbol{\Phi}_{l} \boldsymbol{\delta}_{f(l)}^{*}\right\rangle+\frac{\sigma_{n}^{4}}{\sigma_{s}^{4}} .
\end{aligned}
$$

From (70) and (78), it is clear that proposed ZF and MMSE pairwise equalizers are also efficiently implemented by using FFT.

\section{IUI FREE SUBCARRIER ALLOCATION}

Here, we propose a novel subcarrier mapping method which can significantly suppress the performance degradation due to the Tx IQI for OFDMA and SC-FDMA. In Sec. 2, we show the IUI component in the received signal is the significant source of the performance degradation due to the Tx IQI and also the appearance of the IUI is uniquely determined by the employed subcarrier allocation $\mathbf{M}_{k}$. Therefore, it is possible to choose a certain set of subcarrier assignments in which all the users can avoid the IUI due to their Tx IQIs. Clearly, from (19), if

$$
\beta_{k} \mathbf{\Lambda}_{l k} \mathbf{M}_{l}^{\mathrm{T}} \mathbf{W}^{2} \mathbf{M}_{k} \mathbf{s}_{k}^{*}=\mathbf{0}_{M \times 1}(k \neq l)
$$

holds, there is no IUI due to the Tx IQI. The sufficient condition for this is

$$
\mathbf{M}_{l}^{\mathrm{T}} \mathbf{W}^{2} \mathbf{M}_{k}=\mathbf{0}_{M \times M}(k \neq l) .
$$

Actually, there are many choices for a set of $\mathbf{M}_{k}(k=$ $1, \cdots, K)$ which holds (83) and (11). One simple solution is to assign certain two interleaved allocations for one user based on (30): First we temporarily consider the system with $K^{\prime}=2 K$ users where each user occupies $M / 2$ subcarriers and design $2 K$ interleaved mapping matrices $\mathbf{M}_{k^{\prime}}^{\prime}\left(k^{\prime}=1, \cdots, 2 K\right)$ (Here we assume $K$ and $M$ are power of 2 for simplicity). The resulting IUI only appears between certain users $k^{\prime}$ and $K^{\prime}-k^{\prime}+2$ as in (31). Therefore, in the system with $K$ users, we can derive a set of IUI free $K M \times M$ subcarrier mapping matrices as

$$
\mathbf{M}_{k}=\left\{\begin{array}{ll}
{\left[\begin{array}{ll}
\mathbf{M}_{1}^{\prime} & \mathbf{M}_{K+1}^{\prime}
\end{array}\right]} & (k=1) \\
\mathbf{M}_{k}^{\prime} & \mathbf{M}_{2 K-k+2}^{\prime}
\end{array}\right] \quad\left(\begin{array}{l}
(k \neq 1)
\end{array},\right.
$$

where $k=1, \cdots, K$. An example of subcarrier allocation in the proposed IUI free OFDMA is illustrated in Fig. 4 where $K=4$ and $M=4$.

By the use of such IUI free subcarrier allocations, we can efficiently avoid the performance deterioration due to $\mathrm{Tx}$ IQIs without knowing the imbalance parameters and channel coefficients a priori. This feature is quite attractive in practical systems because the IQ distortion parameter estimation is challenging task when the transceiver suffer from several analog imperfections such as carrier frequency offset or phase noise simultaneously [12], [23].

\section{Simulation Results}

Here, we evaluate the BER performances of OFDMA and SC-FDMA systems with the proposed receivers via computer simulations. In our simulations, we have employed 16QAM with coherent detection for the modulation/demodulation scheme, and set the number of users $K=4$, where all the users are assigned the same transmit power. The number of subcarriers per-user $M=128$, therefore the FFT size is $K M=512$ and the length of $\mathrm{CP}$ is set to be 32 .

The channels and Tx IQIs are randomly generated for each iteration. We considered channels of length $L=10$ with i.i.d circular complex Gaussian coefficients, i.e., $\mathbf{h}_{k} \sim$ $\mathcal{N}_{c}\left(\mathbf{0}_{L \times 1}, \frac{1}{L} \mathbf{I}_{L}\right)(k=1, \cdots K)$. On the other hand, Tx IQI parameters are drawn from uniform distributions, e.g., $\epsilon_{k} \sim \mathcal{U}(0,0.1)$. For the receiver, we test the proposed WL receiver as in Sec. III and the conventional one where user separation is performed as in (19) and the one-tap FDE [1], [3] is employed. All the channel response and IQI parameters are assumed to be known to the receiver and the equalizer weights based on ZF criterion are used both the proposed 
and conventional receiver in OFDMA, while we employ the MMSE weights in SC-FDMA. In all the figures, horizontal axes denotes the received SNR in $\mathrm{dB}$ and vertical axes is the average of per-user BER over 3000 iterations.

First, we test the validity of our analysis. For this purpose, we consider the situation where only the 1st and 2nd users' transmitter suffer from IQI with $\left\{\epsilon_{1}, \epsilon_{2}\right\} \sim \mathcal{U}(0,0.04)$ and $\left\{\theta_{1}, \theta_{2}\right\} \sim \mathcal{U}(-0.04 \pi, 0.04 \pi)$ and the other's have ideal analog front-ends, i.e., $\epsilon_{3}=\epsilon_{4}=\theta_{3}=\theta_{4}=0$. Resulting $I_{R}$ and $I_{2 R}$ are about $35 \mathrm{~dB}$. Fig. 5 shows the user-byuser BER performance of the interleaved OFDMA system with the conventional receiver. From the figure, we can see the significant performance deterioration only on the 4th user. As we have shown in (33), the 4th user's received signal suffer from the IUI due to the 2nd user's image component, meanwhile the image component of the 1st user appears among its own subcarriers. Therefore, the resulting $\operatorname{SIR}_{4}(m)$ ( $m=1, \cdots, M)$ possibly become fatal according to the channel conditions, while $\operatorname{SIR}_{1}(m)=\operatorname{IRR}_{1}$ and the interference of $35 \mathrm{~dB}$ slightly degrade the performance of the $1 \mathrm{st}$ user. Fig. 6 represents the performance of the localized OFDMA with the conventional receiver in the same scenario as Fig. 5. In this case, as in (43), the most of IUI due to the Tx IQIs of the 1st and 2nd transmitter appears on the 4th and 3rd user's received signal respectably. Therefore we can see the significant performance degradation on the 3rd and 4th users.

Next, we show the impact of Tx IQI in the OFDMA with general subcarrier allocation where the IQ distortion occurs at all the transmitters. Here the set of $K$ mapping matrices is randomly generated for each iteration and we set $\left\{\epsilon_{1}, \epsilon_{2}, \epsilon_{3}, \epsilon_{4}\right\} \sim$ $\mathcal{U}(0,0.04)$ and $\left\{\theta_{1}, \theta_{2}, \theta_{3}, \theta_{4}\right\} \sim \mathcal{U}(-0.04 \pi, 0.04 \pi)$. Fig. 7 show the BER performance of the OFDMA with the conventional receiver. In the figure, we also include the performance of OFDM scheme with the conventional ZF equalizer in the same simulation setting for comparison. Recalling that the IRR of $35 \mathrm{~dB}$ is sufficiently small and such Tx IQIs cause a slight performance degradation unless the IUI, the performance of the OFDM receiver deteriorates inconsiderably. On the other hand, in the OFDMA, the IUI possibly occurs between all the users and the BER performances are seriously degraded. Fig. 8 represents the effectiveness the proposed WL ZF receiver in the OFDMA system. From the figure, we can see the significant performance improvement.

On the other hand, the performance of the interleaved SCFDMA or equivalently IFDMA in the presence of the Tx IQI can be seen in Fig. 9 and 10. Fig. 9 shows the BER performance of IFDMA with the conventional receiver with MMSE equalizer weights [19] where we set the Tx IQI parameters as in Fig. 7. From the figure, though we employ the MMSE equalizer, the 2nd and 4th users' BER performance still suffer from error floor, while the 1st and 3rd user can achieve the significant performance improvement due to the diversity gain. Fig. 10 is the performances of the IFDMA system using the proposed pairwise MMSE equalizer. As in Fig. 10, the proposed pairwise equalizer efficiently compensate the interference due to IQI and the channel distortion, and significantly improve the BER performances.

Finally, we test the advantage of the proposed IUI free

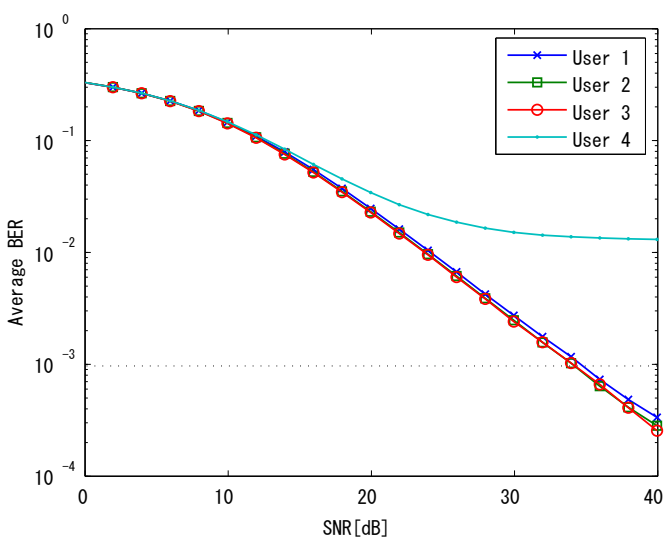

Fig. 5. The BER performance versus SNR of the interleaved OFDMA with the conventional receiver

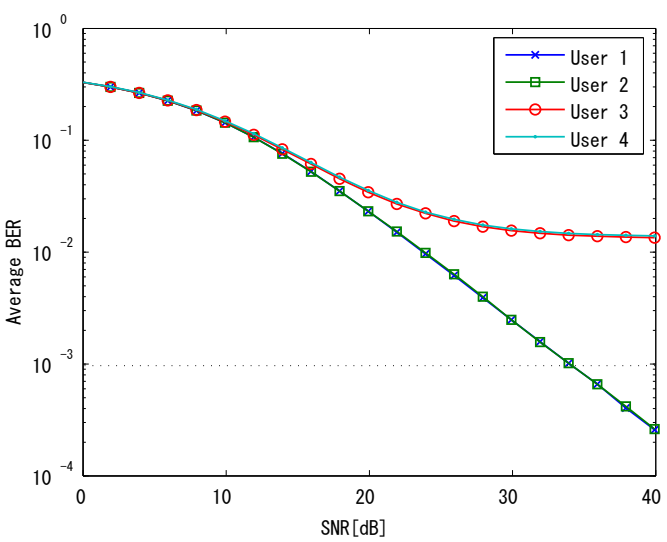

Fig. 6. The BER performance versus SNR of the localized OFDMA with the conventional receiver

subcarrier allocation. In particular, we employ the allocation (84) and the Tx IQI parameters are set the same as in Fig. 7. Fig. 11 shows the performance of OFDMA with the proposed subcarrier allocation and the conventional ZF equalizer. From Fig. 11, by employing the proposed allocation, the received signal efficiently avoid the IUI due to Tx IQIs and achieve the comparable performance to the OFDMA receiver with the proposed WL equalizer. In this case, there is no need to know the Tx IQIs parameters a priori.

\section{CONCLUSION}

In this paper, the effect of the Tx IQIs at the OFDMA and SC-FDMA receiver is investigated. We reveal the IUI due to the IQI is the major source of performance degradation and such IUI emerges depend on the subcarrier mapping used in the system. We proposed the low complexity WL receivers for the OFDMA and SC-FDMA receivers and show the performance improvements with the proposed equalizers via computer simulations. In addition, a novel subcarrier allocation method is proposed to cope with the severe IUI due to the Tx IQ distortion. Under Tx IQIs, we can significantly improve the BER performance of the OFDMA receivers by just employing the proposed mapping. 


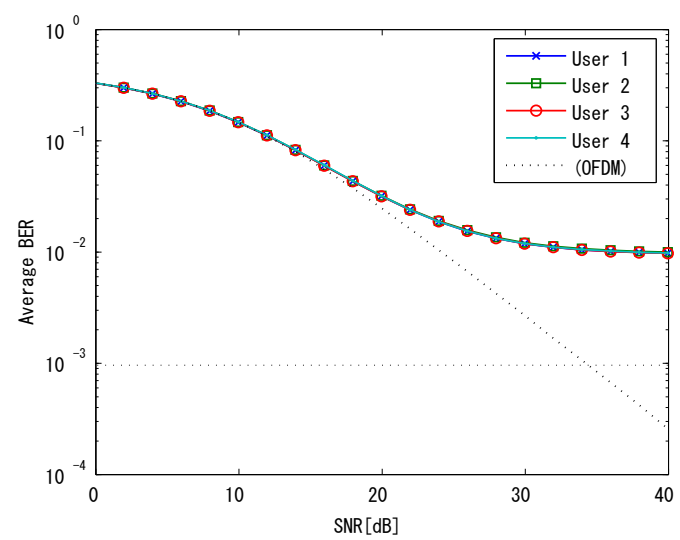

Fig. 7. The BER performance versus SNR of the OFDMA with the randomly generated subcarrier allocation and the conventional receiver

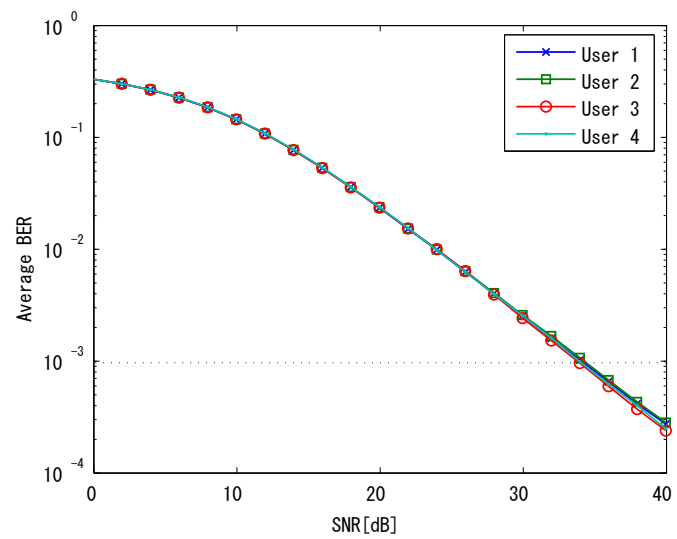

Fig. 8. The BER performance versus SNR of the OFDMA with the randomly generated subcarrier allocation and the proposed WL ZF receiver

\section{REFERENCES}

[1] Z. Wang and G. B. Giannakis, "Wireless multicarrier communications," IEEE Signal Processing Mag., vol. 17, pp. 29-48, May 2000.

[2] IEEE Std. 802.16e, "Air interface for fixed and mobile broadband wireless access systems amendment for physical and medium access control layers for combined fixed and mobile operation in licensed bands," IEEE, 2006

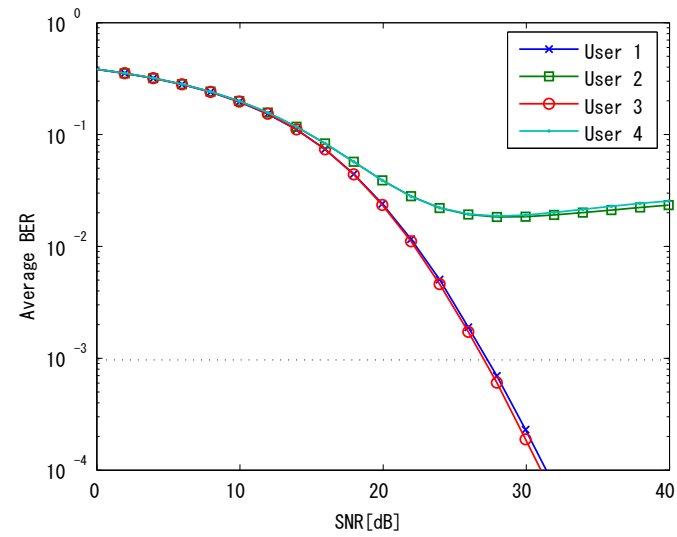

Fig. 9. The BER performance versus SNR of the IFDMA with the conventional receiver based on the MMSE criterion

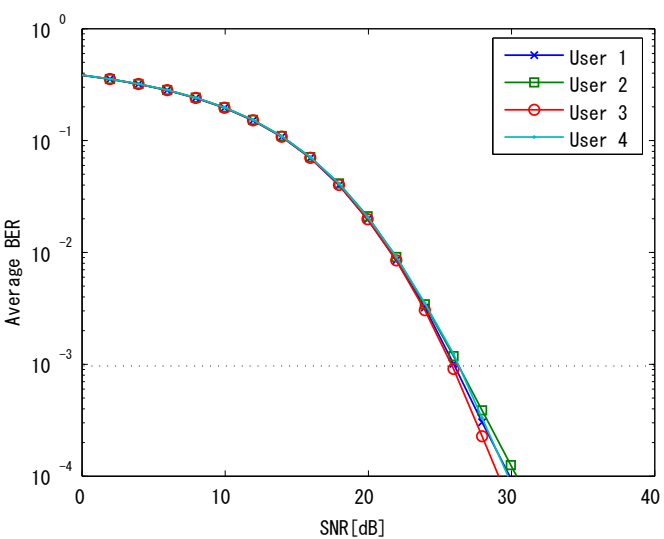

Fig. 10. The BER performance versus SNR of the IFDMA with the proposed WL MMSE receiver

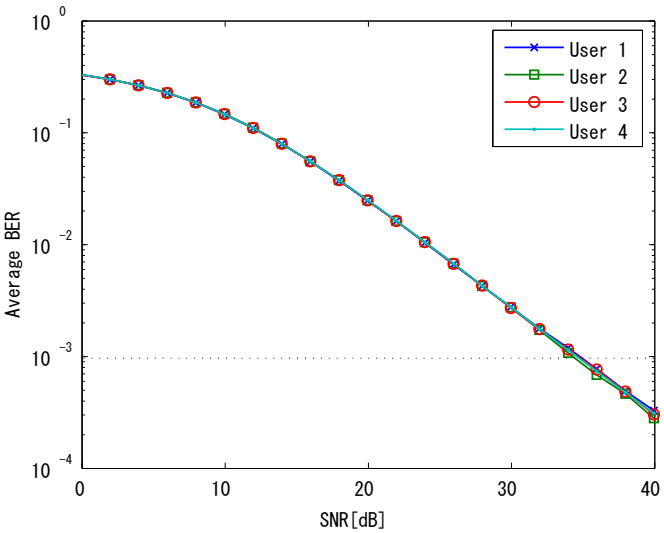

Fig. 11. The BER performance versus SNR of the OFDMA with the proposed IUI free allocation and the conventional receiver

[3] H. Myung, J. Lim, and D. Goodman, "Single Carrier FDMA for Uplink Wireless Transmission," IEEE Vehicular Tech. Mag., vol. 1, no. 3, pp. 30-38, Sep. 2006.

[4] U. Sorger, I. De Broeck, and M. Schnell, "IFDMA - A New Spread Spectrum Multiple Access Scheme," in Proc. of ICC'98, pp. 1013-1017, Atlanta, Georgia, USA, June 1998.

[5] 3GPP TS 36.300, 3rd Generation Partnership Project Technical Specification Group Radio Access Network, "Evolved Universal Terrestrial Radio Access (E-UTRA) and Evolved Universal Terrestrial Radio Access Network (E-UTRAN); Overall description; Stage 2 (Release 8)."

[6] B. Razavi, RF Microelectronics, Prentice Hall, 1998.

[7] C. L. Liu, "Impact of I/Q imbalance on QPSK-OFDM-QAM detection," IEEE Trans. Consumer Electron., vol. 44, no. 8, pp. 984-989, Aug. 1998.

[8] A. Tarighat, R. Bagheri, and A. H. Sayed, "Compensation schemes and performance analysis of IQ imbalances in OFDM receivers," IEEE Trans. Signal Process., vol. 53, no. 8, pp. 3257-3268, Aug. 2005.

[9] J. Tubbax, B. Come, L. Van der Perre, S. Donnay, M. Moonen, and H. De Man, "Compensation of transmitter IQ imbalance for OFDM systems," in Proc. IEEE Int. Conf. Acoust., Speech Signal Process. (ICASSP), pp. 325-328, 2004.

[10] A. Tarighat and A. H. Sayed, "MIMO OFDM receivers for systems with IQ imbalances," IEEE Trans. on Signal Process., vol. 53, no. 9, pp. 3583-3596, 2005.

[11] M. Valkama, M. Renfors, and V. Koivunen, "Blind signal estimation in conjugate signal models with application to I/Q imbalance compensation,” IEEE Signal Process. Lett. , vol. 12, pp. 733-736, Nov. 2005.

[12] J. Tubbax, B. Come, L. V. der Perre, S. Donnay, M. Engels, H. D. Man, and M. Moonen, "Compensation of IQ imbalance and phase noise in OFDM systems," IEEE Trans. Wireless Commun., vol. 4, no. 3, pp. 872-877, May 2005.

[13] M. Lipardi, D. Mattera, and F. Sterle, "MMSE equalization in presence. 
of transmitter and receiver IQ imbalance," in Proc. Int. Waveform Diversity Design Conf. 2007, Pisa, Italy, Jun. 2007, pp. 165-168.

[14] M. Valkama, A. Shahed, L. Anttila, and M. Renfors, "Advanced digital signal processing techniques for compensation of nonlinear distortion in wideband multicarrier radio receivers," IEEE Trans. Microwave Theory and Techniques, vol. 54, pp. 2356-2366, June 2006.

[15] K. Hayashi and H. Sakai, "Interference Cancellation Schemes for Single Carrier Block Transmission with Insufficient Cyclic Prefix," EURASIP Journal on Wireless Communications and Networking, vol. 2008, Article ID 130747, 12 pages, 2008. doi:10.1155/2008/130747

[16] B. Picinbono and P. Chevalier, "Widely linear estimation with complex data," IEEE Trans. Signal Process., vol. 43, pp. 2030-2033, Aug. 1995.

[17] P. Schreier and L. Scharf, "Second-order analysis of improper. complex random vectors and processes," IEEE Trans. Signal Process., vol. 51, no. 3, pp. 714-725, Mar. 2003.

[18] B. Razavi, "Design considerations for direct-conversion receivers," IEEE Trans. Circuits Syst. II, vol. 44, pp. 428-435, June 1997.

[19] D. Falconer, S. L. Ariyavisitakul, A. Benyamin-Seeyar, and B. Eidson, "Frequency Domain Equalization for Single-Carrier Broadband Wireless Systems,” IEEE Commun. Mag., vol. 40, no. 4, pp. 58-66, Apr. 2002.

[20] Y. Yoshida, K. Hayashi and H. Sakai, "Pre- and Post-Equalization and Frequency Diversity Combining Methods for Block Transmission with Cyclic Prefix," IEICE Trans. on Commun., Vol. E90-B, No. 10, pp. 2874-2883, 2007.

[21] D. Darsena, G. Gelli, L. Paura, and F. Verde, "Widely linear equalization and blind channel identification for interference-contaminated multicarrier systems," IEEE Trans. on Signal Proc., vol. 53, no. 3, pp. $1163-1177,2005$.

[22] M. Valkama, M. Renfors, and V. Koivunen, "Compensation of frequency-selective I/Q imbalances in wideband receivers: models and algorithms," in Proc. of the 3rd IEEE Workshop on Signal Processing Advances in Wireless Communications (SPAWC '01), pp. 42-45, Taiwan, Mar. 2001.

[23] D.Tandur and M.Moonen, "Compensation of RF impairments in MIMO OFDM systems", in Proc. of IEEE International Conference on Acoustics, Speech and Signal Processing (ICASSP), pp. 3097-3100, Las Vegas, Apr. 2008.

[24] Y. Yoshida, K. Hayashi, and H. Sakai, "Blind Marginalized Particle Filtering Detector for the Systems with IQ Imbalance and Carrier Frequency Offset," in Proc. of the 16th European Signal Processing Conference (EUSIPCO2008), Lausanne, Switzerland, Aug., 2008.

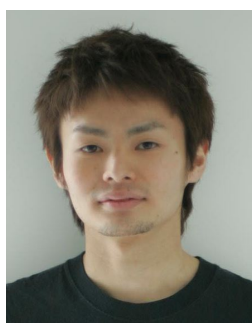

Yuki Yoshida received the B.E. and M. Info. degrees in Systems Science from Kyoto University, Kyoto, Japan, in 2004 and 2005, respectively. He is currently working towards a Ph.D. in the Department of Systems Science, Graduate School of Informatics, Kyoto University.

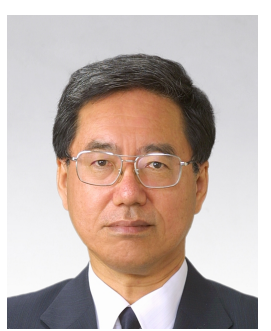

Hideaki Sakai received the B.E. and D.E. degrees in applied mathmatics and physics from Kyoto University, Kyoto, Japan, in 1972 and 1981, respectively. From 1975 to 1978, he was with Tokushima University. He is currently a Professor in the Department of Systems Science, Graduate School of Informatics , Kyoto University. He spent 6 months from 1987 to 1988 at Stanford University as a Visiting Scholar. His research interests are in the areas of adaptive and statistical signal processing. He served as an associated editor of IEEE Trans. Signal Processing from Jan. 1999 to Jan. 2001 and an IEEE Fellow from 2007.

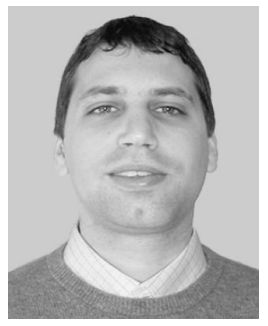

Wladimir Bocquet received the B.E. degree in communication engineering from ENST de Bretagne in 2000, the M.E. degree from University of Rennes in 2000, and Ph.D. in informatics from Kyoto University, Japan in 2008. He joined Fujitsu Laboratories Ltd. in 2000. Since 2005, he has been with France Telecom R

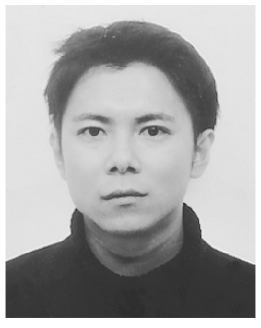

Kazunori Hayashi received the B.E., M.E. and $\mathrm{Ph} . \mathrm{D}$. degrees in communication engineering from Osaka University, Osaka, Japan, in 1997, 1999 and 2002 , respectively. Since 2002, he has been with the Department of System Science Graduate School of Informatics, Kyoto University. He is currently an Assistant Professor there. His research interest include digital signal processing for communications systems. 